\title{
Inflammatory Bowel Diseases: Current Medical and Surgical Therapy
}

\author{
Markus F. Neuratha Ernst Klar ${ }^{\mathrm{b}}$ \\ a Department of Medicine 1, University Erlangen-Nürnberg, Erlangen, Germany, \\ ${ }^{b}$ Department of Surgery, University of Rostock, Rostock, Germany
}

The current edition of ViszeralmEdizin addresses the topic of inflammatory bowel diseases with a special focus on the clinical management of these disorders. Inflammatory bowel diseases comprise Crohn's disease and ulcerative colitis, which are characterized by episodes of abdominal cramping, pain, and chronic diarrhea. The incidence of inflammatory bowel diseases seems to increase in Western countries, and physicians are frequently challenged by the clinical complications of chronic intestinal inflammation in patients with inflammatory bowel diseases. Such complications include the presence of strictures, stenoses, abscess formation, fistulas, and the development of colitis-associated neoplasias and cancer. It is therefore not surprising that inflammatory bowel diseases place a significant burden on the health care systems and are associated with augmented morbidity and health care costs. Finally, these diseases may cause a marked impairment of the quality of life in affected patients.

The early detection and optimized therapy of inflammatory bowel diseases are of utmost importance for the prevention of complications of the disease. These topics are addressed by the invited articles and the interdisciplinary discussion in this issue of Viszeralmedizin. However, many patients still require surgery for the treatment of complications of these disorders. Surgery has to follow certain principles in order to be successful: In Crohn's disease, operative treatment should be performed in a gut-sparing technique and only in the case of complications. In ulcerative coli- tis, pouch surgery is the major challenge, especially if complications occur. By special expertise, control can be achieved in most cases, thus providing a normal quality of life postoperatively. The clinical decision making at the crossroad between internal medicine and surgery is still the subject of an ongoing intense and partially controversial interdisciplinary debate, and this topic is reflected by the interdisciplinary team of authors as well as by the interdisciplinary discussion in this special issue.

Many improvements in the medical therapy of inflammatory bowel diseases have been accomplished in recent years. Starting with the era of anti-TNF (tumor necrosis factor) agents, additional immunosuppressive or anti-inflammatory biological agents blocking proinflammatory cytokines and signaling or adhesion molecules have been developed and tested for the therapy of inflammatory bowel diseases in the context of randomized controlled clinical trials. As highlighted in this issue of Viszeralmedizin, this has led to changes in many of our guidelines for the therapy of inflammatory bowel diseases. Moreover, new surgical techniques have been developed in order to optimize patient care in those affected by inflammatory bowel diseases. It was therefore the vision of the editors to dedicate this issue to an update on the current medical and surgical management of inflammatory bowel diseases. Key gastroenterological and surgical topics are covered by renowned international experts. We hope to provide a stimulating special issue by covering key clinical aspects of this fascinating field.

\section{KARGER \\ Fax +497614520714

Prof. Dr. med. Markus F. Neurath 